\title{
First measurement of the muon neutrino charged current single pion production cross section on water with the T2K near detector
}

K. Abe,${ }^{47}$ C. Andreopoulos,${ }^{45,26}$ M. Antonova, ${ }^{21}$ S. Aoki ${ }^{23}$ A. Ariga, ${ }^{1}$ S. Assylbekov, ${ }^{7}$ D. Autiero, ${ }^{28}$ S. Ban,${ }^{24}$ M. Barbi ${ }^{39}$ G. J. Barker, ${ }^{55}$ G. Barr, ${ }^{35}$ P. Bartet-Friburg, ${ }^{36}$ M. Batkiewicz, ${ }^{12}$ F. Bay, ${ }^{10}$ V. Berardi, ${ }^{17}$ S. Berkman,${ }^{3}$ S. Bhadra, ${ }^{59}$ S. Bienstock ${ }^{36}$ A. Blondel, ${ }^{11}$ S. Bolognesi ${ }^{5}$ S. Bordoni, ${ }^{14}$ S. B. Boyd,${ }^{55}$ D. Brailsford, ${ }^{25,16}$ A. Bravar, ${ }^{11}$ C. Bronner, ${ }^{22}$ M. Buizza Avanzini, ${ }^{9}$ R. G. Calland, ${ }^{22}$ T. Campbell, ${ }^{7}$ S. Cao, ${ }^{24}$ J. Caravaca Rodríguez, ${ }^{14}$

S. L. Cartwright, ${ }^{43}$ R. Castillo, ${ }^{14}$ M. G. Catanesi, ${ }^{17}$ A. Cervera, ${ }^{15}$ D. Cherdack ${ }^{7}$ N. Chikuma, ${ }^{46}$ G. Christodoulou, ${ }^{26}$ A. Clifton, ${ }^{7}$ J. Coleman, ${ }^{26}$ G. Collazuol, ${ }^{19}$ D. Coplowe, ${ }^{35}$ L. Cremonesi, ${ }^{38}$ A. Dabrowska, ${ }^{12}$ G. De Rosa, ${ }^{18}$ T. Dealtry ${ }^{25}$ P. F. Denner, ${ }^{55}$ S. R. Dennis, ${ }^{26}$ C. Densham, ${ }^{45}$ D. Dewhurst,${ }^{35}$ F. Di Lodovico, ${ }^{38}$ S. Di Luise, ${ }^{10}$ S. Dolan, ${ }^{35}$ O. Drapier, ${ }^{9}$ K. E. Duffy ${ }^{35}$ J. Dumarchez ${ }^{36}{ }^{3}$ S. Dytman, ${ }^{37}$ M. Dziewiecki, ${ }^{54}$ S. Emery-Schrenk, ${ }^{5}$ A. Ereditato, ${ }^{1}$ T. Feusels, ${ }^{3}$ A. J. Finch, ${ }^{25}$ G. A. Fiorentini, ${ }^{59}$ M. Friend,,${ }^{13} \dagger$ Y. Fujii,,${ }^{13, \dagger}$ D. Fukuda, ${ }^{33}$ Y. Fukuda, ${ }^{30}$ A. P. Furmanski, ${ }^{55}$ V. Galymov, ${ }^{28}$ A. Garcia, ${ }^{14}$ S. G. Giffin, ${ }^{39}$ C. Giganti, ${ }^{36}$ K. Gilje, ${ }^{32}$ F. Gizzarelli, ${ }^{5}$ M. Gonin, ${ }^{9}$ N. Grant,${ }^{25}$ D. R. Hadley, ${ }^{55}$ L. Haegel, ${ }^{11}$ M. D. Haigh ${ }^{55}$ P. Hamilton, ${ }^{16}$ D. Hansen, ${ }^{37}$ J. Harada,${ }^{34}$ T. Hara, ${ }^{23}$ M. Hartz ${ }^{22,51}$ T. Hasegawa, ${ }^{13, \dagger}$ N. C. Hastings, ${ }^{39}$ T. Hayashino, ${ }^{24}$ Y. Hayato, ${ }^{47,22}$ R. L. Helmer, ${ }^{51}$ M. Hierholzer, ${ }^{1}$ A. Hillairet, ${ }^{52}$ A. Himmel, ${ }^{8}$ T. Hiraki, ${ }^{24}$ S. Hirota, ${ }^{24}$ M. Hogan, ${ }^{7}$ J. Holeczek, ${ }^{44}$ S. Horikawa, ${ }^{10}$ F. Hosomi, ${ }^{46}$ K. Huang ${ }^{24}$ A. K. Ichikawa, ${ }^{24} \mathrm{~K}$. Ieki,${ }^{24}$ M. Ikeda ${ }^{47}$ J. Imber, ${ }^{9}$ J. Insler, ${ }^{27}$ R. A. Intonti, ${ }^{17}$ T. J. Irvine,${ }^{48}$ T. Ishida, ${ }^{13, \dagger}$ T. Ishii, ${ }^{1, \dagger}$ E. Iwai, ${ }^{13}$ K. Iwamoto, ${ }^{40}$ A. Izmaylov, ${ }^{15,21}$ A. Jacob ${ }^{35}$ B. Jamieson, ${ }^{57}$ M. Jiang, ${ }^{24}$ S. Johnson, ${ }^{6}$ J. H. Jo, ${ }^{32}$ P. Jonsson, ${ }^{16}$ C. K. Jung, ${ }^{32, \$}$ M. Kabirnezhad, ${ }^{31}$ A. C. Kaboth, ${ }^{41,45}$ T. Kajita, ${ }^{48,}$ H. Kakuno, ${ }^{49}$ J. Kameda, ${ }^{47}$ D. Karlen ${ }^{52,51}$ I. Karpikov, ${ }^{21}$ T. Katori ${ }^{38}$ E. Kearns, ${ }^{2,22,}$ M. Khabibullin, ${ }^{21}$ A. Khotjantsev, ${ }^{21}$ D. Kielczewska, ${ }^{53, *}$ T. Kikawa, ${ }^{24}$ H. Kim, ${ }^{34}$ J. Kim, ${ }^{3}$ S. King, ${ }^{38}$ J. Kisiel, ${ }^{44}$ A. Knight ${ }^{55}$ A. Knox, ${ }^{25}$ T. Kobayashi, ${ }^{13, \dagger}$ L. Koch, ${ }^{42}$ T. Koga ${ }^{46}$ A. Konaka, ${ }^{51}$ K. Kondo ${ }^{24}$ A. Kopylov, ${ }^{21}$ L. L. Kormos, ${ }^{25}$ A. Korzenev, ${ }^{11}$ Y. Koshio ${ }^{33, \$}$ W. Kropp, ${ }^{4}$ Y. Kudenko, ${ }^{21,8}$ R. Kurjata, ${ }^{54}$ T. Kutter, ${ }^{27}$ J. Lagoda, ${ }^{31}$ I. Lamont ${ }^{25}$ E. Larkin, ${ }^{55}$ P. Lasorak, ${ }^{38,38}$ M. Laveder, ${ }^{19}$ M. Lawe, ${ }^{25}$ M. Lazos, ${ }^{26}$ T. Lindner, ${ }^{51}$ Z. J. Liptak, ${ }^{6}$ R. P. Litchfield ${ }^{16}$ X. Li, ${ }^{32}$ A. Longhin, ${ }^{19}$ J. P. Lopez, ${ }^{6}$ T. Lou, ${ }^{46}$ L. Ludovici, ${ }^{20}$ X. Lu, ${ }^{35}$ L. Magaletti, ${ }^{17}$ K. Mahn, ${ }^{29}$ M. Malek, ${ }^{43}$ S. Manly, ${ }^{40}$ A. D. Marino, ${ }^{6}$ J. Marteau, ${ }^{28}$ J. F. Martin ${ }^{50}$ P. Martins, ${ }^{38}$ S. Martynenko, ${ }^{32}$ T. Maruyama, ${ }^{13, \dagger}$ V. Matveev, ${ }^{21}$ K. Mavrokoridis ${ }^{26}$ W. Y. Ma, ${ }^{16}$ E. Mazzucato, ${ }^{5}$ M. McCarthy, ${ }^{59}$ N. McCauley, ${ }^{26}$ K. S. McFarland,${ }^{40}$ C. McGrew, ${ }^{32}$ A. Mefodiev, ${ }^{21}$ C. Metelko, ${ }^{26}$ M. Mezzetto, ${ }^{19}$ P. Mijakowski, ${ }^{31}$ C. A. Miller ${ }^{51}$ A. Minamino, ${ }^{24}$ O. Mineev ${ }^{21}$ S. Mine, ${ }^{4}$ A. Missert,${ }^{6}$ M. Miura, ${ }^{47, *}$ S. Moriyama, ${ }^{47,}$ Th. A. Mueller, ${ }^{9}$ S. Murphy, ${ }^{10}$ J. Myslik ${ }^{52}$ T. Nakadaira, ${ }^{13, \dagger}$ M. Nakahata, ${ }^{47,22}$ K. G. Nakamura, ${ }^{24}$ K. Nakamura, ${ }^{22,13, \dagger}$ K. D. Nakamura, ${ }^{24}$ S. Nakayama ${ }^{47, \sharp}$ T. Nakaya, ${ }^{24,22}$ K. Nakayoshi, ${ }^{13, \dagger}$ C. Nantais,${ }^{50}$ C. Nielsen, ${ }^{3}$ M. Nirkko, ${ }^{1}$ K. Nishikawa, ${ }^{13, \dagger}$ Y. Nishimura ${ }^{48}$ P. Novella, ${ }^{15}$ J. Nowak, ${ }^{25}$ H. M. O'Keeffe, ${ }^{25}$ R. Ohta, ${ }^{13, \dagger}$

K. Okumura, ${ }^{48,22}$ T. Okusawa, ${ }^{34}$ W. Oryszczak,${ }^{53}$ S. M. Oser,${ }^{3}$ T. Ovsyannikova, ${ }^{21}$ R. A. Owen, ${ }^{38}$ Y. Oyama, ${ }^{13, \dagger}$ V. Palladino, ${ }^{18}$ J. L. Palomino, ${ }^{32}$ V. Paolone, ${ }^{37}$ N. D. Patel, ${ }^{24}$ M. Pavin, ${ }^{36}$ D. Payne, ${ }^{26}$ J. D. Perkin, ${ }^{43}$ Y. Petrov, ${ }^{3}$ L. Pickard ${ }^{43}$ L. Pickering,${ }^{16}$ E. S. Pinzon Guerra, ${ }^{59}$ C. Pistillo, ${ }^{1}$ B. Popov,${ }^{36, \|}$ M. Posiadala-Zezula, ${ }^{53}$

J.-M. Poutissou, ${ }^{51}$ R. Poutissou, ${ }^{51}$ P. Przewlocki, ${ }^{31}$ B. Quilain, ${ }^{24}$ T. Radermacher, ${ }^{42}$ E. Radicioni, ${ }^{17}$ P. N. Ratoff, ${ }^{25}$ M. Ravonel, ${ }^{11}$ M. A. M. Rayner, ${ }^{11}$ A. Redij, ${ }^{1}$ E. Reinherz-Aronis, ${ }^{7}$ C. Riccio ${ }^{18}$ P. Rojas, ${ }^{7}$ E. Rondio, ${ }^{31}$ S. Roth, ${ }^{42}$ A. Rubbia, ${ }^{10}$ A. Rychter ${ }^{54}$ R. Sacco, ${ }^{38}$ K. Sakashita, ${ }^{13,}$ F. Sánchez ${ }^{14}$ F. Sato ${ }^{13}$ E. Scantamburlo, ${ }^{11}$ K. Scholberg, ${ }^{8, *}$ S. Schoppmann, ${ }^{42}$ J. Schwehr, ${ }^{7}$ M. Scott, ${ }^{51}$ Y. Seiya, ${ }^{34}$ T. Sekiguchi, ${ }^{13,{ }^{7}}$ H. Sekiya, ${ }^{47,22, *}$ D. Sgalaberna, ${ }^{11}{ }^{4}$ R. Shah, ${ }^{45,35}$ A. Shaikhiev, ${ }^{21}$ F. Shaker, ${ }^{57}$ D. Shaw ${ }^{25}$ M. Shiozawa, ${ }^{47,22}$ T. Shirahige, ${ }^{33}$ S. Short ${ }^{38}$ M. Smy, ${ }^{4}$

J. T. Sobczyk, ${ }^{58}$ H. Sobel,,${ }^{4,22}$ M. Sorel,${ }^{15}$ L. Southwell, ${ }^{25}$ P. Stamoulis, ${ }^{15}$ J. Steinmann, ${ }^{42}$ T. Stewart, ${ }^{45}$ P. Stowell, ${ }^{43}$ Y. Suda, ${ }^{46}$ S. Suvorov, ${ }^{21}$ A. Suzuki, ${ }^{23}$ K. Suzuki, ${ }^{24}$ S. Y. Suzuki, ${ }^{13, \dagger}$ Y. Suzuki, ${ }^{22}$ R. Tacik,${ }^{39,51}$ M. Tada, ${ }^{13, \dagger}$ S. Takahashi, ${ }^{24}$ A. Takeda, ${ }^{47}$ Y. Takeuchi, ${ }^{23,22}$ H. K. Tanaka, ${ }^{47,}$ H. A. Tanaka, ${ }^{50,51,9}$ D. Terhorst, ${ }^{42}$ R. Terri, ${ }^{38}$ T. Thakore, ${ }^{27}$ L. F. Thompson, ${ }^{43}$ S. Tobayama,${ }^{3}$ W. Toki, ${ }^{7}$ T. Tomura, ${ }^{47}$ C. Touramanis, ${ }^{26}$ T. Tsukamoto, ${ }^{13, \dagger}$ M. Tzanov, ${ }^{27}$ Y. Uchida, ${ }^{16}$ A. Vacheret ${ }^{16}$ M. Vagins, ${ }^{22,4}$ Z. Vallari, ${ }^{32}$ G. Vasseur, ${ }^{5}$ T. Wachala, ${ }^{12}$

K. Wakamatsu, ${ }^{34}$ C. W. Walter, ${ }^{8,}$ D. Wark, ${ }^{45,35}$ W. Warzycha, ${ }^{53}$ M. O. Wascko, ${ }^{16,13}$ A. Weber, ${ }^{45,35}$ R. Wendell, ${ }^{24, \dagger}$ R. J. Wilkes, ${ }^{56}$ M. J. Wilking, ${ }^{32}$ C. Wilkinson, ${ }^{1}$ J. R. Wilson, ${ }^{38}$ R. J. Wilson, ${ }^{7}$ Y. Yamada, ${ }^{13, \dagger}$ K. Yamamoto, ${ }^{34}$ M. Yamamoto, ${ }^{24}$ C. Yanagisawa, ${ }^{32, * *}$ T. Yano, ${ }^{23}$ S. Yen, ${ }^{51}$ N. Yershov, ${ }^{21}$ M. Yokoyama, ${ }^{46, *}$ J. Yoo, ${ }^{27}$

K. Yoshida, ${ }^{24}$ T. Yuan, ${ }^{6}$ M. Yu, ${ }^{59}$ A. Zalewska, ${ }^{12}$ J. Zalipska, ${ }^{31}$ L. Zambelli, ${ }^{13, \dagger}$ K. Zaremba, ${ }^{54}$ M. Ziembicki, ${ }^{54}$ E. D. Zimmerman, ${ }^{6}$ M. Zito, ${ }^{5}$ and J. Żmuda ${ }^{58}$

(T2K Collaboration)

\footnotetext{
${ }^{1}$ University of Bern, Albert Einstein Center for Fundamental Physics, Laboratory for High Energy Physics (LHEP), Bern, Switzerland ${ }^{2}$ Boston University, Department of Physics, Boston, Massachusetts, USA
} 
${ }^{3}$ University of British Columbia, Department of Physics and Astronomy, Vancouver, British Columbia, Canada

${ }^{4}$ University of California, Irvine, Department of Physics and Astronomy, Irvine, California, USA ${ }^{5}$ IRFU, CEA Saclay, Gif-sur-Yvette, France

${ }^{6}$ University of Colorado at Boulder, Department of Physics, Boulder, Colorado, USA

${ }^{7}$ Colorado State University, Department of Physics, Fort Collins, Colorado, USA

${ }^{8}$ Duke University, Department of Physics, Durham, North Carolina, USA

${ }^{9}$ Ecole Polytechnique, IN2P3-CNRS, Laboratoire Leprince-Ringuet, Palaiseau, France

${ }^{10}$ ETH Zurich, Institute for Particle Physics, Zurich, Switzerland

${ }^{11}$ University of Geneva, Section de Physique, DPNC, Geneva, Switzerland

${ }^{12} \mathrm{H}$. Niewodniczanski Institute of Nuclear Physics PAN, Cracow, Poland

${ }^{13}$ High Energy Accelerator Research Organization (KEK), Tsukuba, Ibaraki, Japan

${ }^{14}$ Institut de Fisica d'Altes Energies (IFAE), The Barcelona Institute of Science and Technology, Campus UAB, Bellaterra. Barcelona, Spain

${ }^{15}$ IFIC (CSIC \& University of Valencia), Valencia, Spain

${ }^{16}$ Imperial College London, Department of Physics, London, United Kingdom

${ }^{17}$ INFN Sezione di Bari and Università e Politecnico di Bari, Dipartimento Interuniversitario di Fisica, Bari, Italy

${ }^{18}$ INFN Sezione di Napoli and Università di Napoli, Dipartimento di Fisica, Napoli, Italy

${ }^{19}$ INFN Sezione di Padova and Università di Padova, Dipartimento di Fisica, Padova, Italy

${ }^{20}$ INFN Sezione di Roma and Università di Roma "La Sapienza”, Roma, Italy

${ }^{21}$ Institute for Nuclear Research of the Russian Academy of Sciences, Moscow, Russia

${ }^{22}$ Kavli Institute for the Physics and Mathematics of the Universe (WPI),

The University of Tokyo Institutes for Advanced Study, University of Tokyo, Kashiwa, Chiba, Japan

${ }^{23}$ Kobe University, Kobe, Japan

${ }^{24}$ Kyoto University, Department of Physics, Kyoto, Japan

${ }^{25}$ Lancaster University, Physics Department, Lancaster, United Kingdom

${ }^{26}$ University of Liverpool, Department of Physics, Liverpool, United Kingdom

${ }^{27}$ Louisiana State University, Department of Physics and Astronomy, Baton Rouge, Louisiana, USA

${ }^{28}$ Université de Lyon, Université Claude Bernard Lyon 1, IPN Lyon (IN2P3), Villeurbanne, France

${ }^{29}$ Michigan State University, Department of Physics and Astronomy, East Lansing, Michigan, USA

${ }^{30}$ Miyagi University of Education, Department of Physics, Sendai, Japan

${ }^{31}$ National Centre for Nuclear Research, Warsaw, Poland

${ }^{32}$ State University of New York at Stony Brook, Department of Physics and Astronomy, Stony Brook, New York, USA

${ }^{33}$ Okayama University, Department of Physics, Okayama, Japan

${ }^{34}$ Osaka City University, Department of Physics, Osaka, Japan

${ }^{35}$ Oxford University, Department of Physics, Oxford, United Kingdom

${ }^{36}$ UPMC, Université Paris Diderot, CNRS/IN2P3, Laboratoire de Physique Nucléaire et de Hautes Energies (LPNHE), Paris, France

${ }^{37}$ University of Pittsburgh, Department of Physics and Astronomy, Pittsburgh, Pennsylvania, USA

${ }^{38}$ Queen Mary University of London, School of Physics and Astronomy, London, United Kingdom

${ }^{39}$ University of Regina, Department of Physics, Regina, Saskatchewan, Canada

${ }^{40}$ University of Rochester, Department of Physics and Astronomy, Rochester, New York, USA

${ }^{41}$ Royal Holloway University of London, Department of Physics, Egham, Surrey, United Kingdom

${ }^{42}$ RWTH Aachen University, III. Physikalisches Institut, Aachen, Germany

${ }^{43}$ University of Sheffield, Department of Physics and Astronomy, Sheffield, United Kingdom

${ }^{44}$ University of Silesia, Institute of Physics, Katowice, Poland

${ }^{45}$ STFC, Rutherford Appleton Laboratory, Harwell Oxford, and Daresbury Laboratory,

Warrington, United Kingdom

${ }^{46}$ University of Tokyo, Department of Physics, Tokyo, Japan

${ }^{47}$ University of Tokyo, Institute for Cosmic Ray Research, Kamioka Observatory, Kamioka, Japan

${ }^{48}$ University of Tokyo, Institute for Cosmic Ray Research, Research Center for Cosmic Neutrinos, Kashiwa, Japan

${ }^{49}$ Tokyo Metropolitan University, Department of Physics, Tokyo, Japan

${ }^{50}$ University of Toronto, Department of Physics, Toronto, Ontario, Canada

${ }^{51}$ TRIUMF, Vancouver, British Columbia, Canada

${ }^{52}$ University of Victoria, Department of Physics and Astronomy, Victoria, British Columbia, Canada

${ }^{53}$ University of Warsaw, Faculty of Physics, Warsaw, Poland 


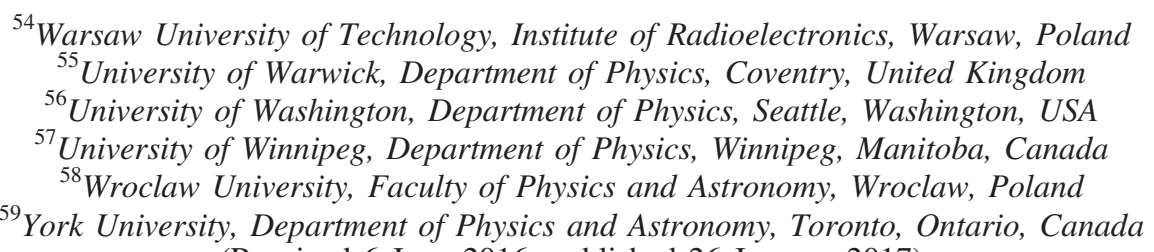
(Received 6 June 2016; published 26 January 2017)

\begin{abstract}
The T2K off-axis near detector, ND280, is used to make the first differential cross section measurements of muon neutrino charged current single positive pion production on a water target at energies $\sim 0.8 \mathrm{GeV}$. The differential measurements are presented as a function of the muon and pion kinematics, in the restricted phase space defined by $p_{\pi^{+}}>200 \mathrm{MeV} / c, p_{\mu}>200 \mathrm{MeV} / c, \cos \left(\theta_{\pi^{+}}\right)>0.3$ and $\cos \left(\theta_{\mu}\right)>0.3$. The total flux integrated $\nu_{\mu}$ charged current single positive pion production cross section on water in the restricted phase space is measured to be $\langle\sigma\rangle_{\phi}=4.25 \pm 0.48$ (stat) \pm 1.56 (syst) $\times 10^{-40} \mathrm{~cm}^{2} /$ nucleon. The total cross section is consistent with the NEUT prediction $\left(5.03 \times 10^{-40} \mathrm{~cm}^{2} /\right.$ nucleon $)$ and $2 \sigma$ lower than the GENIE prediction $\left(7.68 \times 10^{-40} \mathrm{~cm}^{2} /\right.$ nucleon $)$. The differential cross sections are in good agreement with the NEUT generator. The GENIE simulation reproduces well the shapes of the distributions, but overestimates the overall cross section normalization.
\end{abstract}

DOI: 10.1103/PhysRevD.95.012010

\section{INTRODUCTION}

The T2K long baseline neutrino experiment [1] has the primary goal to precisely measure neutrino oscillation parameters through measurements of $\nu_{e}$ appearance and $\nu_{\mu}$ disappearance from a $\nu_{\mu}$ beam. As neutrinos are chargeless and colorless, neutrino oscillation experiments rely on the detection of charged particles coming from charged current (CC) and neutral current (NC) interactions to infer neutrino properties, e.g. CC quasielastic (QE) interactions allow the calculation of the neutrino energy from the lepton kinematics. The knowledge of $\nu_{\mu}$ and $\nu_{e}$ interaction cross sections is then fundamental to infer neutrino properties correctly. $\nu_{\mu} \mathrm{CC}$ resonant interactions are part of the signal and sometimes of the background of oscillation experiments, and a better understanding of this channel could be beneficial not only to $\mathrm{T} 2 \mathrm{~K}$, but to the neutrino community in general, as there are discrepancies between models and experimental data.

Both the MiniBooNE [2] and MINERvA [3] collaborations provided measurements of the $\mathrm{CC}$ single positive pion

\footnotetext{
*Deceased.

Also at J-PARC, Tokai, Japan.

${ }^{\ddagger}$ Also at Kavli IPMU (WPI), the University of Tokyo, Japan.

§Also at National Research Nuclear University " MEPhI" and Moscow Institute of Physics and Technology, Moscow, Russia.

"Also at JINR, Dubna, Russia.

"Also at Institute of Particle Physics, Canada.

Also at BMCC/CUNY, Science Department, New York, New York, USA.

Published by the American Physical Society under the terms of the Creative Commons Attribution 3.0 License. Further distribution of this work must maintain attribution to the author(s) and the published article's title, journal citation, and DOI.
}

production $\left(\mathrm{CC} 1 \pi^{+}\right)$cross sections in mineral oil and plastic scintillator, respectively. The $\mathrm{CC} 1 \pi^{+}$cross section is described by the particles leaving the nucleus, i.e. one muon, one positive pion and any number of nucleons. There are large discrepancies between the MiniBooNE and MINERvA experiments, and the historic ANL [4] and BNL [5] bubble chamber results, which could be due to nuclear effects that if not modeled correctly can modify the effective measured cross section. The MiniBooNE and MINERvA results show also significant normalization and shape discrepancies between each other [3], and currently no theoretical model can explain all the pion production data available. Additional pion production data can help to constrain the pion production models and give valuable information on the nucleon- $\Delta$ axial form factor $[6,7]$.

We present the first $\mathrm{CC} 1 \pi^{+}$differential cross section measurements on water. $\mathrm{A} \mathrm{CC} 1 \pi^{+}$measurement on water will have a strong impact on the $\mathrm{T} 2 \mathrm{~K}$ oscillation analysis, as current results suffer from large cross section systematic uncertainties related to the differences in targets between near and far detectors (carbon versus water) [8]. These data will also be beneficial to future atmospheric and long-baseline experiments, which plan to use a water target, such as the Hyper-Kamiokande experiment [9].

\section{T2K EXPERIMENT}

The T2K long baseline neutrino experiment uses the J-PARC facility in Tokai, Japan, to produce $30 \mathrm{GeV}$ protons, which produce charged pions by colliding with a graphite target and consequently result in a high purity $\nu_{\mu}$ beam. The beam center axis is directed $2.5^{\circ}$ off axis towards 
Super-Kamiokande [10] at $295 \mathrm{~km}$ from J-PARC. Two near detectors are located at $280 \mathrm{~m}$ from the target, the onaxis near detector (INGRID [11]) and the off-axis near detector (ND280).

\section{A. Neutrino beam flux}

The predicted neutrino beam flux [12] peaks at $0.6 \mathrm{GeV}$ and its fractional composition is $92.6 \% \nu_{\mu}, 6.2 \% \bar{\nu}_{\mu}, 1.1 \%$ $\nu_{e}, 0.1 \% \bar{\nu}_{e}$. The proton interactions with the graphite target are simulated with the FLUKA2008 package [13], The propagation of secondary and tertiary pions and kaons and their decays to neutrinos is simulated with GEANT3 [14]. The hadron interactions are modeled with GCALOR [15] and tuned to hadron production data from external experiments, such as the CERN NA61/SHINE experiment [16-18].

\section{B. Neutrino interaction model}

Based on the prediction of the neutrino flux, the NEUT [19] (version 5.1.4.2) event generator is used to simulate neutrino interactions in ND280.

For charged current quasielastic (CCQE) and neutral current quasielastic interactions, NEUT uses the Llewellyn Smith model [20] integrated with the relativistic Fermi gas model by Smith and Moniz to describe the nucleons within the nucleus [21]. The outgoing nucleon is also required to have larger momentum than the Fermi surface momentum (Pauli blocking), which is $217 \mathrm{MeV} / c$ for carbon and $225 \mathrm{MeV} / c$ for oxygen.

NEUT uses the Rein-Sehgal model for resonant interactions [22], considering 18 resonances with masses below $2 \mathrm{GeV} / c^{2}$ and their interference terms. In addition $20 \%$ of the $\Delta$ resonances undergo pionless $\Delta$ decay, in which the $\Delta$ is absorbed by the nuclear medium without emitting any pions: $\Delta+N \rightarrow N^{\prime}+N^{\prime \prime}$. The NEUT pion production model is tuned using neutrino interaction data from the MiniBooNE experiments [2,23], as explained in Ref. [8]. In particular, the axial mass for resonant $\mathrm{CC} 1 \pi^{+}$interactions is set to $1.41 \mathrm{GeV}$, and the overall $\mathrm{CC} 1 \pi^{+}$normalization for energies less than $2.5 \mathrm{GeV}$ is further increased by $15 \%$ compared to predictions when the axial mass is set to $1.41 \mathrm{GeV}$.

Coherent pion production is simulated for both $\mathrm{NC}$ and CC interactions using the Rein-Sehgal model [24], including the partially conserved axial vector current lepton mass correction for CC interactions [25].

Deep inelastic scattering (DIS) processes are simulated using GRV98 parton distribution functions [26] and corrections following the Bodek and Yang model [27] to improve the agreement with experiments in the low- $Q^{2}$ region. To avoid double counting with the single pion resonant production, only multiple pion production processes are considered for the invariant mass of the recoiling hadron system $W<2 \mathrm{GeV} / c^{2}$. PYTHIA/JETSET [28] is used for hadronization at energies above $2 \mathrm{GeV}$, and an internal NEUT method is used at lower energies.

After the simulation of the initial neutrino-nucleon interaction, final state interactions are simulated with the cascade model [29]. Each particle is propagated inside the nucleus with steps determined by the mean-free path. The mean-free path depends on the position inside the nucleus and the momentum of the particle. At each step, an interaction is generated according to the probability calculated from each cross section such as charge exchange, absorption or scattering. If an interaction occurs, the resulting particles are used for stepping through the rest of the nucleus. This process continues until all particles are either absorbed in the nucleus or escape it. Data from several pion scattering experiments are used to tune this model [8].

Additional information on the models used to simulate the neutrino interactions and the hadron transport in the nuclear medium can be found in Refs. [8,19].

The results in this paper are also compared to the GENIE generator [30], as it provides a general framework valid over a large range of experiments, targets and neutrino energy. GENIE uses essentially the same models as NEUT for the neutrino interactions simulation, but they differ in the implementation and value of some of the parameters, such as $M_{A}^{R E S}$ which is set to $1.12 \mathrm{GeV}$ [31]. Single pion production (before final state interactions) comes from resonant and coherent processes in NEUT, whereas GENIE also considers DIS contributions to it. Although GENIE considers a lower value of $M_{A}^{R E S}$, the predicted single positive pion production cross section is larger than in NEUT, because DIS processes are allowed to contribute to this state.

\section{Near detector}

ND280 is a complex of different subdetectors enclosed in the refurbished UA1/NOMAD magnet. The origin of the ND280 coordinate system is at the center of the magnet and the $0.2 \mathrm{~T}$ magnetic field is along the $+x$ direction. The $z$ axis is along the nominal neutrino beam axis, and $x$ and $y$ axes are horizontal and vertical, respectively.

The ND280 Tracker region contains two fine-grained detectors (FGDs [32]) which are used as the neutrino interaction target, sandwiched between three gaseous time projection chambers (TPCs [33]) which are used to track charged particles. The most upstream FGD (FGD1) primarily consists of polystyrene scintillator bars with layers oriented alternately in the $x$ and $y$ directions allowing 3D tracking of charged particles. The downstream FGD (FGD2) has a similar structure, but the polystyrene bars are interleaved with water layers, creating a modular structure of water layer $+x$ layer $+y$ layer + water layer, and so on (see Fig. 1). The areal density of an $x y$ module and a water module are respectively $2146.3 \pm 14.4 \mathrm{mg} / \mathrm{cm}^{2}$ and $2792.6 \pm 13.4 \mathrm{mg} / \mathrm{cm}^{2}$. This 
structure allows the measurement of neutrino interactions on water.

The electromagnetic calorimeters (ECals [34]), made of layers of lead and scintillator bars, surround the Tracker region (Barrel-ECals) with one module downstream of it (Ds-ECal). Upstream of the Tracker there is a $\pi^{0}$ detector (PØD [35]), consisting of scintillator, water and brass layers.

Magnet return yokes surround the entire detector to make the magnetic field uniform and contain it inside the detector. Plastic scintillators in the yoke form the side muon range detectors [36].

The analysis here presented uses FGD2 as the active interaction target, where in a signal event the neutrino interacts with a nucleus in the water layer, and the charged lepton coming from a CC interaction is tracked in the downstream scintillator layers. These results are based on data taken from November 2010 to May 2013. The good quality data collected during this period corresponds to $5.6 \times 10^{20}$ protons on target.

\section{SELECTION OF $\nu_{\mu} \mathrm{CC} 1 \pi^{+}$ INTERACTIONS IN WATER}

Muon neutrino interactions are selected by using the highest momentum negative track starting in the fiducial volume (FV) of the FGD2. The FGD2 FV begins $58 \mathrm{~mm}$ inward from the lateral edges of the FGD2 and $7.5 \mathrm{~mm}$ inward from the upstream FGD2 edge (as shown in Fig. 1).

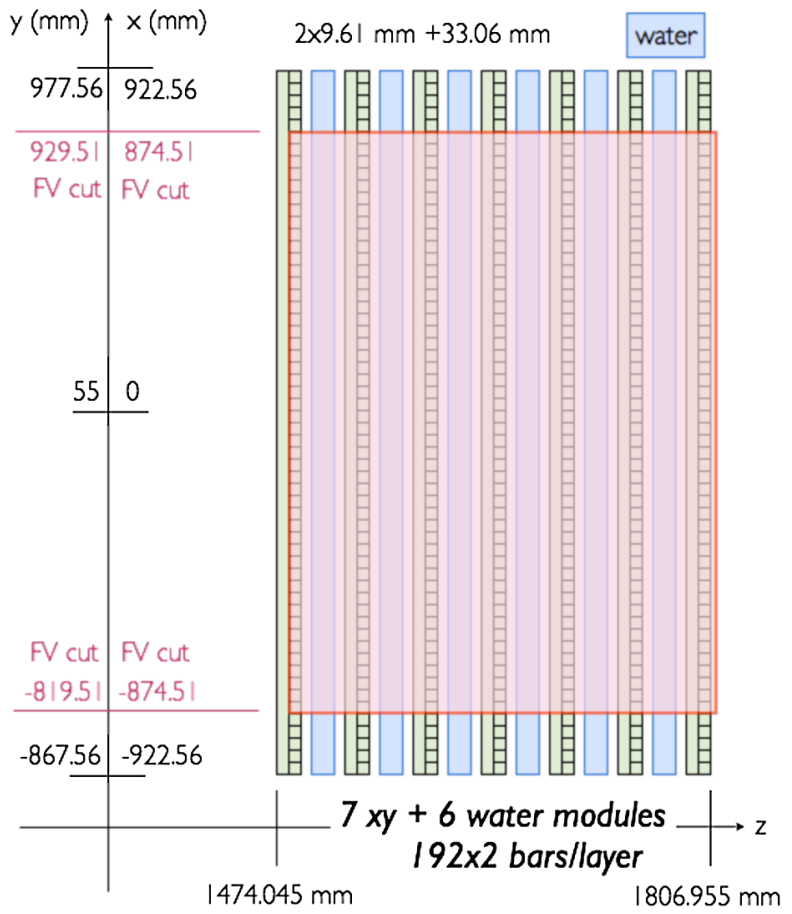

FIG. 1. Schematic view of FGD2 and its fiducial volume (FV) delimited by the red line. The first upstream scintillator layer is not included in the FV.
These tracks are required to enter the TPC3 (located immediately downstream of FGD2) and deposit energy compatible with a muonlike track. Additional tracks matched between the FGD and TPC associated with the same muon candidate vertex are tagged as either protons or positive, negative or neutral pions by looking at the trajectory and energy deposit in the TPCs, and at electromagnetic showers in the ECals. More details on the $\nu_{\mu} \mathrm{CC}$ inclusive and multipion selections can be found in Refs. [8,37], respectively, where the only differences are that in this analysis interactions in FGD2 are selected, rather than in FGD1, and the ECals are used to tag neutral pions. $\mathrm{CC} 1 \pi^{+}$-like events are selected by requiring one muon, one positive pion, no other additional pions and any number of nucleons.

Because of the structure of the FGD2 (see Fig. 1), interaction vertices occurring in a water module will be reconstructed in the $x$ layer downstream of it. A waterenhanced sample can be selected by requiring the vertex to be in the $x$ layer, whilst a scintillator sample can be selected by requiring the reconstructed vertex to be in the $y$ layer.

The signal sample of this analysis is composed of 1402 selected $\mathrm{CC} 1 \pi^{+}$water-enhanced events in the full phase space, with $30.9 \%$ purity of true $\nu_{\mu} \mathrm{CC} 1 \pi^{+}$interactions on water. To avoid relying on the simulation to describe regions of efficiency $<0.1$, the analysis presented restricts the kinematic phase space to the region defined by $p_{\mu}>200 \mathrm{MeV} / c, \quad p_{\pi^{+}}>200 \mathrm{MeV} / c, \quad \cos \left(\theta_{\mu}\right)>0.3$ and $\cos \left(\theta_{\pi^{+}}\right)>0.3$. With these restrictions in the phase space of the signal definition, the signal efficiency goes from $13.3 \%$ to $30.7 \%$.

The signal sample is selected with a purity of $39.9 \%$ in the restricted phase space. Multipion interactions can be misidentified as $\mathrm{CC} 1 \pi^{+}$interactions when one or more pions are absorbed by the detector or simply not reconstructed; $29.2 \%$ of the signal sample is composed of this background. $\mathrm{CC} 0 \pi$ interactions $(3.9 \%)$ come into the selection when the proton is misidentified as a $\pi^{+}$. The total background from $\mathrm{CC} 1 \pi^{+}$interactions occurring in the scintillator amounts to $25.7 \%$ of the signal sample, including interactions occurring in the $y$ layer whose vertex is reconstructed in the $x$ layer. Non- $\nu_{\mu}$ CC interactions $(0.6 \%)$ include both NC and CC interactions due to the $\bar{\nu}$ and $\nu_{e}$ components in the beam. They mainly come into the selection when a $\pi^{-}$from a NC interaction is misidentified as the muon candidate.

These backgrounds are constrained with two external samples. A sample of selected $\nu_{\mu} \mathrm{CC}$ events with one $\pi^{+}$ and at least one, but maximum 3 , negative or neutral pions $\left(\mathrm{CC} 1 \pi^{+} \mathrm{n} \pi\right.$ water-enhanced sample) is used to constrain the non-CC $1 \pi^{+}$interactions, which include the CC non- $1 \pi^{+}$, non- $\nu_{\mu} \mathrm{CC}$ and out of $\mathrm{FV}$ backgrounds. A sample of selected $\nu_{\mu} \mathrm{CC} 1 \pi^{+}$events in the $y$ layers of the FGD2 $\left(\mathrm{CC} 1 \pi^{+}\right.$scintillator sample) is used to constrain the background coming from the interactions in the scintillator. 
TABLE I. Percentage of true NEUT topologies $\left(\mathrm{CC} 1 \pi^{+}\right.$and CC-non- $\left.1 \pi^{+}\right)$in the restricted phase space, and number of data events in different modules of the FGD2 for the signal $\left(\mathrm{CC} 1 \pi^{+}\right.$ water enhanced) and two external samples $\left(\mathrm{CC} 1 \pi^{+}\right.$scintillator and $\mathrm{CC} 1 \pi^{+} \mathrm{n} \pi$ water enhanced).

\begin{tabular}{lccc}
\hline \hline & \multicolumn{3}{c}{ Selected samples } \\
\cline { 2 - 4 } True NEUT & $\begin{array}{c}\mathrm{CC} 1 \pi^{+} \\
\text {water }\end{array}$ & $\begin{array}{c}\mathrm{CC} 1 \pi^{+} \\
\text {scintillator }\end{array}$ & $\begin{array}{c}\mathrm{CC} 1 \pi^{+} \mathrm{n} \pi \\
\text { water }\end{array}$ \\
\hline $\mathrm{CC} 1 \pi^{+}$water & $39.9 \%$ & $5.9 \%$ & $7.7 \%$ \\
$\mathrm{CC} 1 \pi^{+}$scintillator & $25.7 \%$ & $54.6 \%$ & $4.8 \%$ \\
$\mathrm{CC}$ non- $1 \pi^{+}$water & $18.5 \%$ & $8.3 \%$ & $49.0 \%$ \\
$\mathrm{CC}$ non- $1 \pi^{+}$scintillator & $14.6 \%$ & $28.7 \%$ & $36.5 \%$ \\
non- $\nu_{\mu} \mathrm{CC}$ & $0.6 \%$ & $0.9 \%$ & $1.2 \%$ \\
Out of FV & $0.5 \%$ & $1.7 \%$ & $0.9 \%$ \\
Data in reduced phase & 1275 & 431 & 885 \\
$\quad$ space & & & \\
Data in full phase space & 1402 & 491 & 944 \\
\hline \hline
\end{tabular}

Table I shows the composition of the signal and external samples according to the NEUT generator. Distributions of the pion kinematics $\left[p_{\pi^{+}}\right.$and $\left.\cos \left(\theta_{\pi^{+}}\right)\right]$, the muon kinematics $\left[p_{\mu}\right.$ and $\left.\cos \left(\theta_{\mu}\right)\right]$, the cosine of the angle between the muon and the pion $\left[\cos \left(\theta_{\left(\mu, \pi^{+}\right)}\right)\right]$and reconstructed neutrino energy $\left(E_{\nu}^{\text {rec }}\right)$ in the selected sample are shown in Fig. 2. The reconstructed neutrino energy is found by applying 4-momentum conservation and assuming the target nucleon is at rest and the remaining final-state particle is a nucleon:

$$
E_{\nu}^{\mathrm{rec}}=\frac{m_{\mu}^{2}+m_{\pi}^{2}-2 m_{N} E_{f}+2\left(p_{\mu} \cdot p_{\pi}\right)}{\left(2 E_{f}-\mathbf{p}_{\mu} \cos \left(\theta_{\mu}\right)-\mathbf{p}_{\pi} \cos \left(\theta_{\pi^{+}}\right)-m_{N}\right)},
$$

where $m_{\mu}, m_{\pi}$ and $m_{N}$ are the masses of the muon, the pion and the nucleon respectively; $E_{f}=E_{\mu}+E_{\pi} ; p_{x}, \mathbf{p}_{\mathrm{x}}$ and $\theta_{x}$ are the 4-momentum, 3-momentum and angle with the neutrino direction of the particle considered $\left(x=\mu, \pi^{+}\right)$. Distributions of the pion momentum in the external samples are found in Fig. 3.

\section{A. Systematic uncertainties}

The TPC and FGD detector systematic uncertainties are the same as the ones described in Refs. [8,37]. The ECal particle identification systematic uncertainties are evaluated with high purity samples of electrons and muons, as explained in Ref. [38].

The isolated ECal reconstruction systematic uncertainty is evaluated with a control sample of both isolated and nonisolated ECal objects, due to the difficulties of finding a control sample with just isolated ECal objects. The efficiency is found to be $0.303 \pm 0.003$ in simulation and $0.315 \pm 0.009$ in data for the Barrel-ECal, and $0.826 \pm$ 0.002 in simulation and $0.839 \pm 0.007$ for the Ds-ECal. These efficiencies are used to correct the simulation efficiency for tagging isolated-ECal objects only, which is 0.352 for the Barrel-ECal and 0.163 for the Ds-ECal.

The FGD water modules mass uncertainty is $0.55 \%$. The FGD layer migration uncertainties have been evaluated in detail for this analysis. These migrations are divided into forward (i.e. when the reconstructed vertex is a layer downstream of the true vertex) and backward migrations (i.e. when the reconstructed vertex is a layer upstream of the true vertex). The forward migrations come from a hit reconstruction inefficiency. Their overall uncertainty is estimated to be $3.3 \%$ with a control sample of cosmic muons passing through both FGDs. The backward migrations come from low energy backward going particles that are fitted with the muon track and move the vertex one or more layers upstream. These latter migrations are estimated using the $\mathrm{CC} 0 \pi$ and $\mathrm{CC}$ multipion samples in FGD2: a normalization uncertainty of $30 \%$ is assigned to them.

The flux uncertainties are evaluated with beam line and hadron production measurements. The hadron production uncertainties dominate the neutrino flux uncertainties, with a smaller contribution from the neutrino beam direction and proton beam uncertainties. The systematic uncertainty for the $\nu_{\mu}$ flux at ND280 varies from 10\% and 15\% depending on the neutrino energy [12].

The uncertainties related to the cross section model (final state interactions, CCQE model, pion production model and nuclear model) are constrained using external data and comparisons between different existing models. A summary of these uncertainties can be found in Ref. [8].

\section{UNFOLDING METHOD}

The Bayesian unfolding technique by d'Agostini [39] has been successfully used by past T2K cross section measurements to extract the cross sections (see Refs. [37,40]). The first estimate of the true distribution is found by applying the unsmearing matrix $P\left(t_{j} \mid r_{i}\right)$ (found with Bayes' theorem) to the data distribution:

$$
\hat{N}_{t_{j}}=\frac{1}{\epsilon_{j}} \sum_{i} P\left(t_{j} \mid r_{i}\right)\left(N_{r_{i}}-\sum_{k}^{\text {all backgrounds }} \alpha_{k} B_{r_{i}, k}\right),
$$

where $t_{j}\left(r_{i}\right)$ indicates the true (reconstructed) bin for each observable, $N_{r_{i}}$ is the number of reconstructed events in bin $r_{i}, B_{r_{i}, k}$ is the number of predicted events in bin $r_{i}$ of background type $k, \alpha_{k}$ is a normalization constant derived from the external samples, and $\epsilon_{j}$ is the true efficiency in bin $t_{j}$.

Equation (2) uses a background subtraction where the coefficients $\alpha_{k}$ are 1 if that part of the background is not constrained by any external sample, or otherwise calculated as 

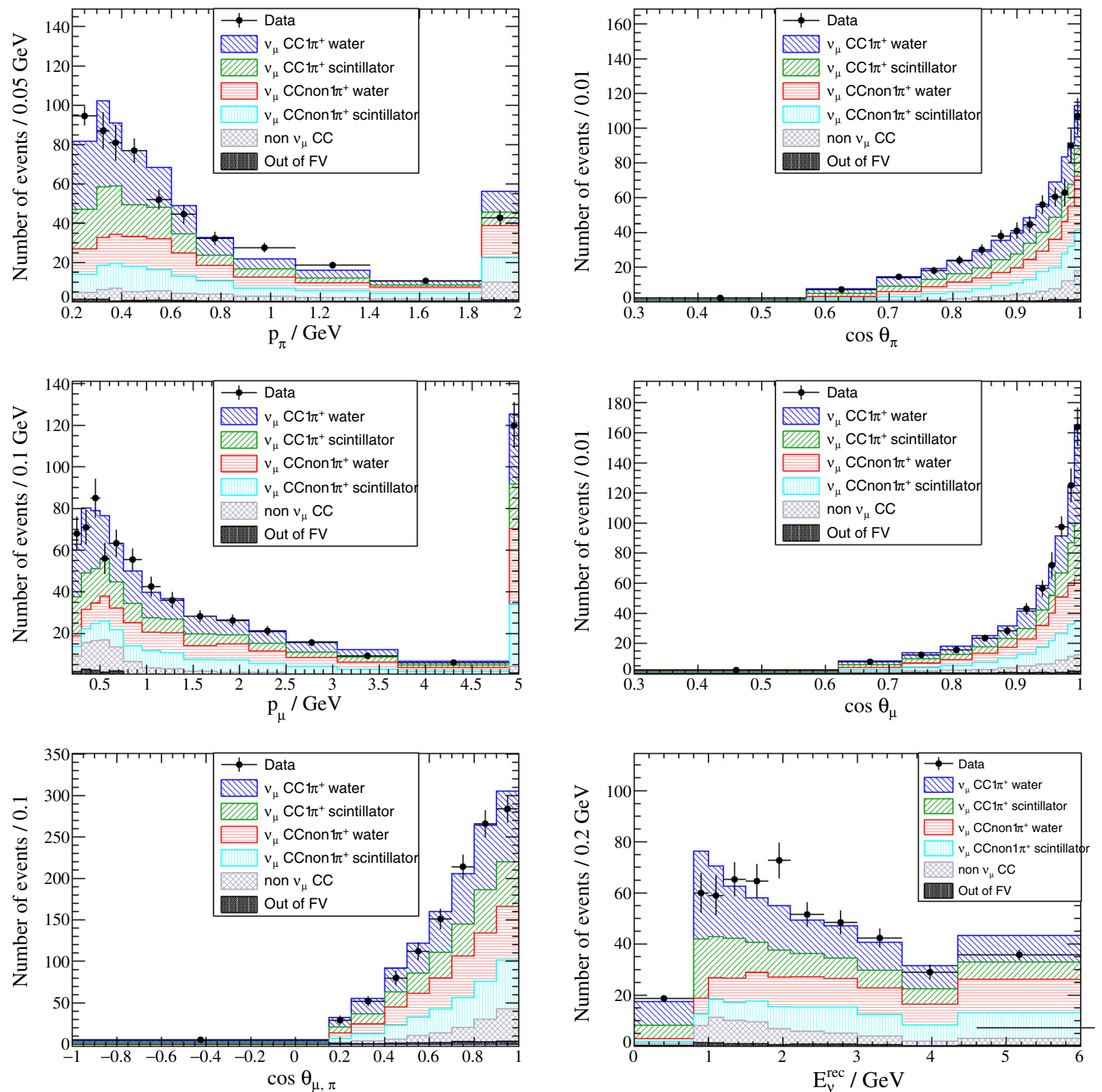

FIG. 2. Reconstructed pion kinematics (top), muon kinematics (middle), $\cos \left(\theta_{\left(\mu, \pi^{+}\right)}\right)$(bottom left) and neutrino energy (bottom right) distributions of the events in the $\nu_{\mu} \mathrm{CC} 1 \pi^{+}$water-enhanced sample. The NEUT Monte Carlo prediction is separated into the $\nu_{\mu} \mathrm{CC} 1 \pi^{+}$ interactions in water, $\nu_{\mu} \mathrm{CC} 1 \pi^{+}$interactions in scintillator, $\nu_{\mu}$ CC-non- $1 \pi^{+}$interactions in water, $\nu_{\mu}$ CC-non- $1 \pi^{+}$interactions in scintillator, non- $\nu_{\mu}$ CC interactions, and interactions outside of the FGD2 FV. The last bin in the $p_{\pi^{+}}, p_{\mu}$ and $E_{\nu}^{\text {rec }}$ distributions contains all the overflow events.

$$
\alpha_{k}=\frac{C_{\mathrm{data}, k}}{C_{\mathrm{MC}, k}},
$$

where $C_{\text {data, } k}$ is the total number of events in external sample $k$ in data and $C_{\mathrm{MC}, k}$ is the total number of events in external sample $k$ in MC. In this analysis the background is divided into two groups: the $\mathrm{CC} 1 \pi^{+}$interactions in the scintillator or in the vessels which enclose the water and have a similar composition to the scintillator plane (scintillator-line components), which are constrained with the $\mathrm{CC} 1 \pi^{+}$scintillator sample; the non-CC $1 \pi^{+}$background which is constrained with the $\mathrm{CC} 1 \pi^{+} \mathrm{n} \pi$ water-enhanced sample.

The FGD2 water modules are composed of oxygen (73.83\%), carbon $(15.05 \%)$, hydrogen $(10.48 \%)$, silicon $(0.39 \%)$, and magnesium $(0.25 \%)$. The carbon, silicon and magnesium come from the polycarbonate structure that enclose the liquid water. They compose the scintillatorlike component of the water modules and can be subtracted out with the $x$-layer as they have similar composition.

The effect of the systematic uncertainties on the cross section measurements is evaluated by using pseudo-experiments. For each pseudo-experiment the signal and control 

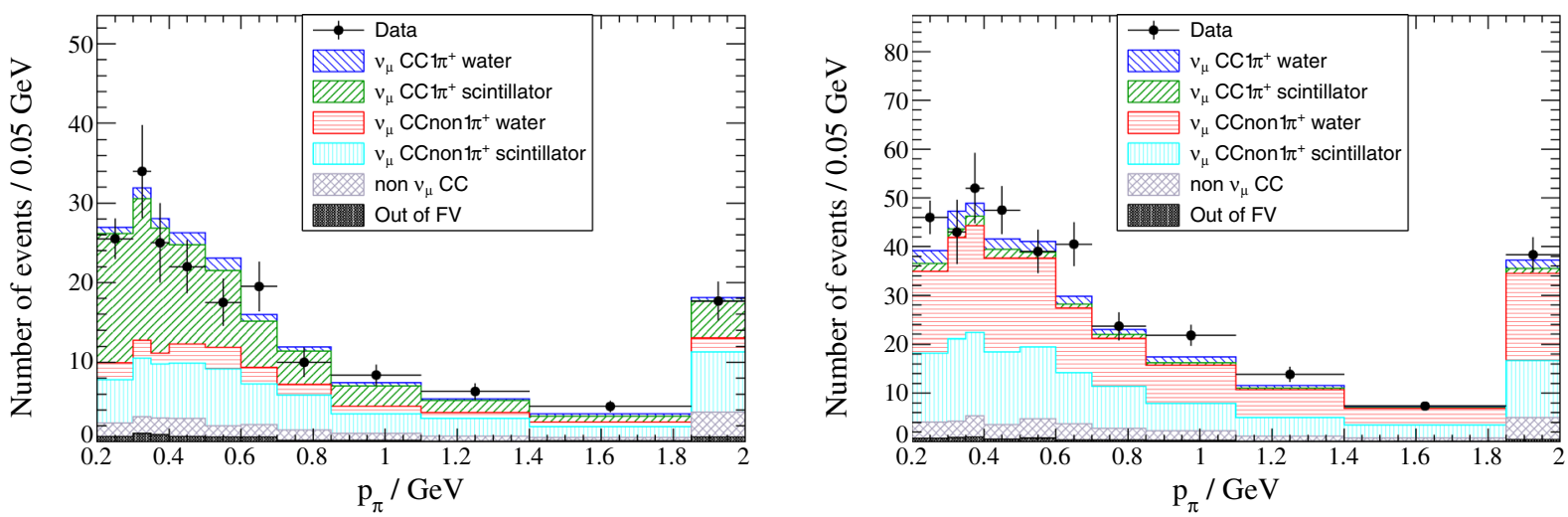

FIG. 3. Reconstructed pion momentum distributions of the events in the two external samples: CC1 $\pi^{+} \operatorname{scintillator}\left(\right.$ left) and CC $1 \pi^{+} \mathrm{n} \pi$ water-enhanced (right). The last bin contains all the overflow events.

samples are smeared according to the error source considered, the normalization constants are reevaluated and used to renormalize the signal prediction before evaluating the cross section for that throw. The covariance matrix is then defined as

$$
V_{i j}^{s}=\frac{1}{N} \sum_{s_{n}=1}^{N}\left(\sigma_{i}^{s_{n}}-\sigma_{i}^{\mathrm{nom}}\right)\left(\sigma_{j}^{s_{n}}-\sigma_{j}^{\mathrm{nom}}\right),
$$

where $\sigma_{i}^{s_{n}}$ is the differential cross section in bin $i$ evaluated with throw $n$ of the uncertainty $s$, and $\sigma_{i}^{\text {nom }}$ is the nominal differential cross section in bin $i$. Statistical and systematic uncertainties are evaluated by varying the contents of each bin according to Poisson and Gaussian statistics, respectively.

\section{CROSS-SECTION RESULTS}

For a given variable $X$, the flux integrated differential cross section for bin $t_{k}$ is defined as

$$
\left\langle\frac{\partial \sigma}{\partial X}\right\rangle_{t_{k}}=\frac{N_{t_{k}}^{\text {unfolded }}}{T \Phi \Delta X_{t_{k}}}
$$

where $N_{t_{k}}^{\text {unfolded }}$ is the estimated number of events in bin $t_{k}$ [as given by Eq. (2)], $T$ is the number of target nucleons, $\Phi$ is the $\nu_{\mu}$ flux per unit area and integrated over neutrino energy (as detailed in Ref. [12]), and $\Delta X_{t_{k}}$ is the width of bin $t_{k}$. Even though single pion resonant production has a threshold at $480 \mathrm{MeV}$, no cut is applied to the $\nu_{\mu}$ flux, as the $\mathrm{CC} 1 \pi^{+}$signal definition includes processes with different thresholds as well.

The number of target nucleons is computed considering only the oxygen and hydrogen in the FGD2 water modules, as the carbon, silicon and magnesium components are removed by the Bayesian unfolding with background subtraction. The total number of target nucleons is found to be

$$
T=N_{A} \cdot V_{\mathrm{FV}} \cdot \rho \sum_{a=\mathrm{O}, \mathrm{H}} f_{a} \frac{A_{a}}{M_{a}}=2.55 \times 10^{29},
$$

where $N_{A}=6.022 \times 10^{23} \mathrm{~mol}^{-1}$ is the Avogadro number, $V_{\mathrm{FV}}$ is the volume of the modules considered inside the $\mathrm{FV}, \rho=\rho_{\text {area }} / \Delta z$ is the total density of the water modules of the FGD2 $\left(\rho_{\text {area }}=2798.7 \pm 5.4 \mathrm{mg} / \mathrm{cm}^{2}\right.$ is the total areal density and $\Delta z=2.79 \mathrm{~cm}$ is the width of each water module); $a$ runs over the elements present in the water modules; $f_{a}$ is the mass fraction; $A_{a}$ represents the averaged number of nucleons per nucleus; and $M_{a}$ is the atomic mass.

The normalization constant found from the $\mathrm{CC} 1 \pi^{+}$ control region is $0.989 \pm 0.050$ indicating that the number of scintillator interactions is compatible with the prediction from the simulation. The normalization constant related to the non-CC $1 \pi^{+}$background is $1.104 \pm 0.039$ indicating that the non-CC $1 \pi^{+}$interactions are slightly more in data than simulation. These two constants are used to renormalize the background, and hence constrain the systematic uncertainties.

Figure 4 shows the differential cross section as a function of pion kinematics (top), muon kinematics (center), $\cos \left(\theta_{\left(\mu, \pi^{+}\right)}\right)$(bottom left), and $E_{\nu}^{\text {rec }}$ (bottom right). For the $E_{\nu}^{\text {rec }}$ the $\sigma(E)$ is presented as a model dependent result, as the $E_{\nu}^{\text {rec }}$ is unfolded to the true neutrino energy as predicted by NEUT. The NEUT and GENIE (version 2.6.4 [30]) predictions are also shown in the plots. The differential cross sections obtained are compatible with the NEUT prediction, but a small suppression is seen at $0.5<$ $p_{\pi^{+}}<0.7 \mathrm{GeV}$ and $\cos \left(\theta_{\pi^{+}}\right)>0.95$. This might be linked to the model for $\mathrm{CC}$ coherent interactions used in NEUT: NEUT greatly overestimates the amount of coherent interactions especially at low $E_{\pi^{+}}$[41]. The GENIE simulation reproduces well the shapes of the distributions, but overestimates the overall cross section normalization. 

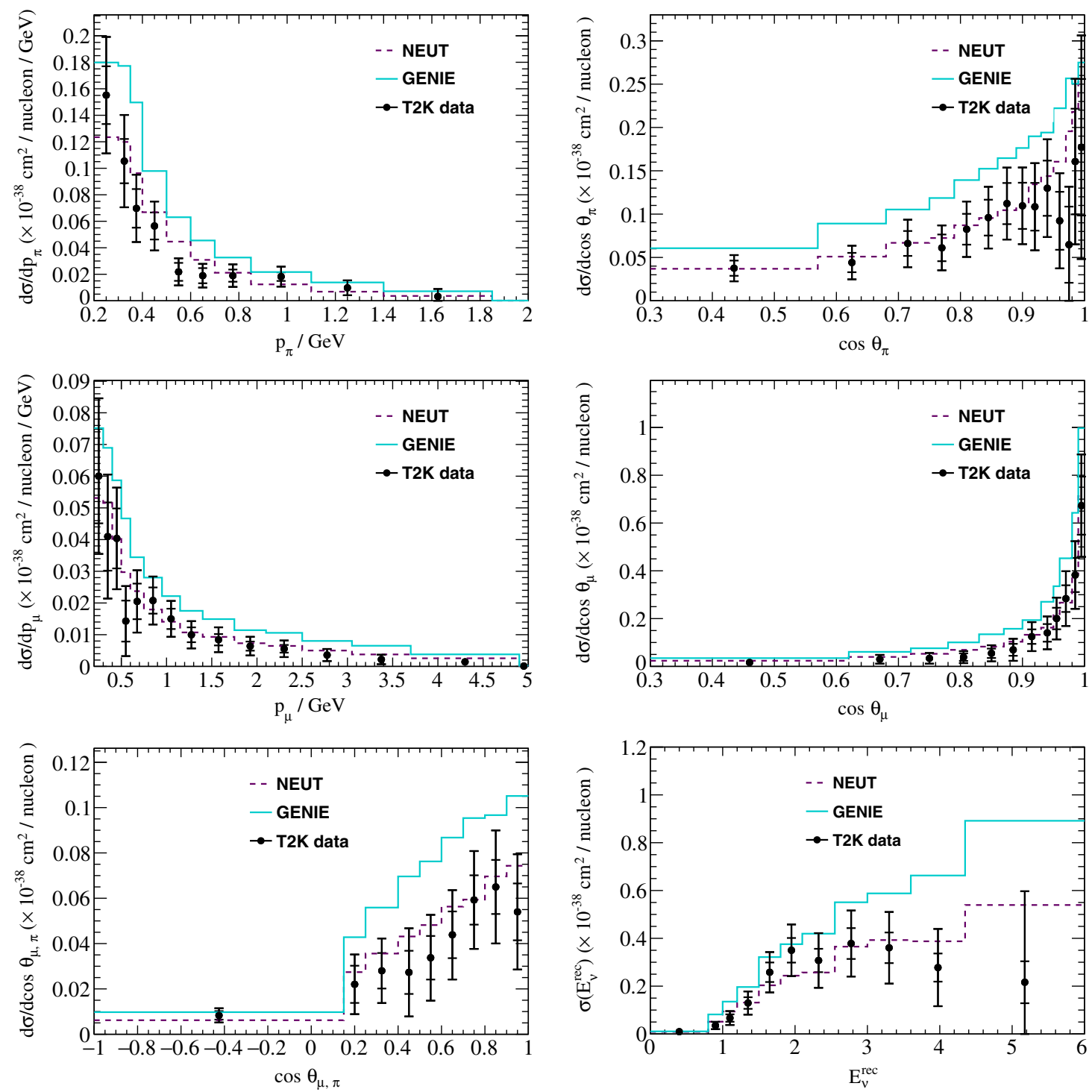

FIG. 4. Unfolded $\nu_{\mu} \mathrm{CC} 1 \pi^{+}$differential cross sections as a function of pion kinematics (top), muon kinematics (center), $\cos \left(\theta_{\left(\mu, \pi^{+}\right)}\right)$ (bottom left) and $E_{\nu}^{\text {rec }}$ (bottom right) in the reduced phase space of $p_{\pi^{+}}>200 \mathrm{MeV} / c, p_{\mu}>200 \mathrm{MeV} / c, \cos \left(\theta_{\pi^{+}}\right)>0.3$ and $\cos \left(\theta_{\mu}\right)>0.3$. For the $E_{\nu}^{\mathrm{rec}}$, the $\sigma(E)$ is presented as a model dependent result. The inner (outer) error bars show the statistical (total) uncertainty on the data. The dashed (solid) line shows the NEUT, version 5.1.4.2, (GENIE, version 2.6.4) prediction.

The total flux integrated cross section is computed as

$$
\langle\sigma\rangle_{\Phi}=\frac{N_{\text {total }}}{T \cdot \Phi}
$$

The total flux integrated $\nu_{\mu} \mathrm{CC}$ single positive pion production cross section on water in the restricted phase space is measured to be $\langle\sigma\rangle_{\phi}=4.25 \pm 0.48$ (stat) \pm 1.56 (syst) $\times 10^{-40} \mathrm{~cm}^{2} /$ nucleon. This result is compatible with the NEUT prediction of $5.03 \times 10^{-40} \mathrm{~cm}^{2} /$ nucleon, and about $2 \sigma$ away from the GENIE prediction $7.68 \times 10^{-40} \mathrm{~cm}^{2} /$ nucleon. The dominant systematic uncertainties on this result are those related to the cross section model (23.9\%) and flux parameters (25.5\%), because of the low purity of the selected signal sample. Without the selected control samples both these uncertainties would be as high as $40 \%$. Nonetheless the low statistics and purity of the selected control samples makes it difficult to further reduce these uncertainties. Final state interactions and detector systematic uncertainties contribute with $5.3 \%$ and $10.8 \%$, respectively. The data and MC statistical errors are estimated as $10.7 \%$ and $3.3 \%$, respectively. Figure 5 shows the total $\nu_{\mu} \mathrm{CC} 1 \pi^{+}$cross section on water in the reduced phase space of $p_{\pi^{+}}>200 \mathrm{MeV} / c$, $p_{\mu}>200 \mathrm{MeV} / c, \cos \left(\theta_{\pi^{+}}\right)>0.3$ and $\cos \left(\theta_{\mu}\right)>0.3$, with the T2K $\nu_{\mu}$ flux and the NEUT and GENIE predictions.

Future analyses will consider the use of the FGD2 and FGD1 samples simultaneously, eliminating the necessity to 


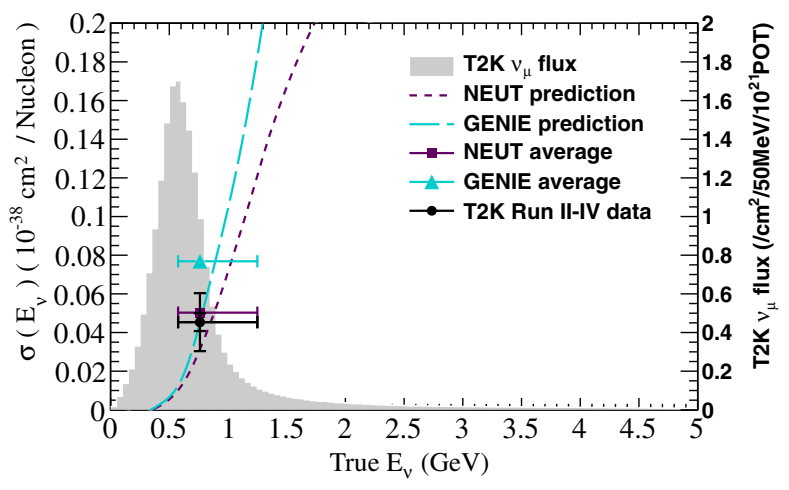

FIG. 5. Total $\nu_{\mu} \mathrm{CC} 1 \pi^{+}$cross section on water in the reduced phase space of $p_{\pi^{+}}>200 \mathrm{MeV} / c, p_{\mu}>200 \mathrm{MeV} / c$, $\cos \left(\theta_{\pi^{+}}\right)>0.3$ and $\cos \left(\theta_{\mu}\right)>0.3$. The T2K data point is placed at the $\nu_{\mu}$ flux mean energy. The vertical error represents the total uncertainty, and the horizontal bar represents $68 \%$ of the flux each side of the mean. The T2K flux distribution is shown in gray. The NEUT (version 5.1.4.2) and GENIE (version 2.6.4) predictions are the total $\nu_{\mu} \mathrm{CC} 1 \pi^{+}$predictions as a function of neutrino energy. The NEUT and GENIE averages are the fluxaveraged predictions.

divide the FGD2 sample in the $x$ and $y$ layers and allowing the simultaneous evaluation of the cross sections on scintillator and water. This technique will considerably reduce both the flux and theory cross-section uncertainties that currently limit this measurement.

The data related to this measurement can be found together with the cross section results obtained when unfolding the muon kinematics and neutrino energy distributions in Ref. [42].

\section{CONCLUSION}

The T2K off-axis near detector ND280 is used to extract the first $\nu_{\mu} \mathrm{CC} 1 \pi^{+}$differential cross sections on water as a function of the pion kinematics and muon-pion angle. These results will be beneficial to the T2K experiment and the neutrino community in general, as a better understanding of neutrino induced pion production on water at energy below $2 \mathrm{GeV}$ would result in a higher sensitivity to the measurement of oscillation parameters. The cross section is evaluated in the restricted phase space defined by $p_{\mu}>200 \mathrm{MeV} / c, p_{\pi^{+}}>200 \mathrm{MeV} / c, \cos \left(\theta_{\mu}\right)>0.3$ and $\cos \left(\theta_{\pi^{+}}\right)>0.3$. The results are in good agreement with the NEUT generator and a general suppression is seen compared to the GENIE generator. The total $\nu_{\mu}$ $\mathrm{CC} 1 \pi^{+}$cross section on water is found to be $\langle\sigma\rangle_{\phi}=$ $4.25 \pm 0.48$ (stat) \pm 1.56 (syst) $\times 10^{-40} \mathrm{~cm}^{2} /$ nucleon, which is in good agreement with the NEUT prediction and is $2 \sigma$ lower than the GENIE prediction.

\section{ACKNOWLEDGMENTS}

We thank the J-PARC staff for superb accelerator performance and the CERN NA61 collaboration for providing valuable particle production data. We acknowledge the support of MEXT, Japan; NSERC (Grant No. SAPPJ2014-00031), NRC and CFI, Canada; CEA and CNRS/ IN2P3, France; DFG, Germany; INFN, Italy; National Science Centre (NCN), Poland; RSF, RFBR and MES, Russia; MINECO and ERDF funds, Spain; SNSF and SERI, Switzerland; STFC, UK; and DOE, USA. We also thank CERN for the UA1/NOMAD magnet, DESY for the HERA-B magnet mover system, NII for SINET4, the WestGrid and SciNet consortia in Compute Canada, and GridPP in the United Kingdom. In addition, participation of individual researchers and institutions has been further supported by funds from ERC (FP7), H2020 Grant No. RISE-GA644294-JENNIFER, EU; JSPS, Japan; Royal Society, UK; and the DOE Early Career program, USA.
[1] K. Abe et al. (T2K Collaboration), Nucl. Instrum. Methods Phys. Res., Sect. A 659, 106 (2011).

[2] A. A. Aguilar-Arevalo et al. (MiniBooNE Collaboration), Phys. Rev. D 83, 052007 (2011).

[3] B. Eberly et al. (Minerva Collaboration), Phys. Rev. D 92 , 092008 (2015).

[4] G. M. Radecky et al., Phys. Rev. D 25, 1161 (1982).

[5] T. Kitagaki et al., Phys. Rev. D 34, 2554 (1986).

[6] O. Lalakulich and U. Mosel, Phys. Rev. C 88, 017601 (2013).

[7] M. Martini and M. Ericson, Phys. Rev. C 90, 025501 (2014).

[8] K. Abe et al. (T2K Collaboration), Phys. Rev. D 91, 072010 (2015).
[9] K. Abe et al. (Hyper-Kamiokande Collaboration), Prog. Theor. Exp. Phys. 2015, 053C02 (2015).

[10] S. Fukuda et al. (Super-Kamiokande Collaboration), Nucl. Instrum. Methods Phys. Res., Sect. A 501, 418 (2003).

[11] M. Otani et al., Nucl. Instrum. Methods Phys. Res., Sect. A 623, 368 (2010).

[12] K. Abe et al. (T2K Collaboration), Phys. Rev. D 87, 012001 (2013).

[13] G. Battistoni, F. Cerutti, A. Fasso, A. Ferrari, S. Muraro, J. Ranft, S. Roesler, and P. Sala, in AIP Conf. Proc. 896, 31 (2007).

[14] R. Brun, F. Carminati, and S. Giani, Report No. CERNW5013, 1994. 
[15] C. Zeitnitz and T. A. Gabriel, in Proceedings of International Conference on Calorimetry in High Energy Physics, Tallahassee, FL, USA, February 1993 (World Scientific, Singapore, 2014).

[16] N. Abgrall et al. (NA61/SHINE Collaboration), Phys. Rev. C 84, 034604 (2011).

[17] N. Abgrall et al. (NA61/SHINE Collaboration), Phys. Rev. C 85, 035210 (2012).

[18] N. Abgrall et al. (NA61/SHINE Collaboration), Eur. Phys. J. C 76, 84 (2016).

[19] Y. Hayato, Nucl. Phys. B, Proc. Suppl. 112, 171 (2002).

[20] C. L. Smith, Phys. Rep. 3, 261 (1972).

[21] R. Smith and E. Moniz, Nucl. Phys. B43, 605 (1972).

[22] D. Rein and L. M. Sehgal, Ann. Phys. (N.Y.) 133, 79 (1981).

[23] A. A. Aguilar-Arevalo et al. (MiniBooNE Collaboration), Phys. Rev. D 83, 052009 (2011).

[24] D. Rein and L. M. Sehgal, Nucl. Phys. B223, 29 (1983).

[25] D. Rein and L. Sehgal, Phys. Lett. B 657, 207 (2007).

[26] M. Glück, E. Reya, and A. Vogt, Eur. Phys. J. C 5, 461 (1998).

[27] A. Bodek and U. Yang, arXiv:hep-ex/0308007.

[28] T. Sjöstrand, Comput. Phys. Commun. 82, 74 (1994).

[29] L. Salcedo, E. Oset, M. Vicente-Vacas, and C. Garcia-Recio, Nucl. Phys. A484, 557 (1988).
[30] C. Andreopoulos et al., Nucl. Instrum. Methods Phys. Res., Sect. A 614, 87 (2010).

[31] K. S. Kuzmin, V. V. Lyubushkin, and V. A. Naumov, Acta Phys. Pol. B 37, 2337 (2006).

[32] P. Amaudruz et al., Nucl. Instrum. Methods Phys. Res., Sect. A 696, 1 (2012).

[33] N. Abgrall et al., Nucl. Instrum. Methods Phys. Res., Sect. A 637, 25 (2011).

[34] D. Allan et al., J. Instrum. 8, P10019 (2013).

[35] S. Assylbekov et al., Nucl. Instrum. Methods Phys. Res., Sect. A 686, 48 (2012).

[36] S. Aoki et al., Nucl. Instrum. Methods Phys. Res., Sect. A 698, 135 (2013).

[37] K. Abe et al. (T2K Collaboration), Phys. Rev. D 87, 092003 (2013).

[38] K. Abe et al. (T2K Collaboration), Phys. Rev. D 89, 092003 (2014).

[39] G. D’Agostini, Nucl. Instrum. Methods Phys. Res., Sect. A 362, 487 (1995).

[40] K. Abe et al. (T2K Collaboration), Phys. Rev. Lett. 113, 241803 (2014).

[41] K. Abe et al. (T2K Collaboration), Phys. Rev. Lett. 117, 192501 (2016).

[42] K. Abe et al., T2K public data, http://t2k-experiment.org/ results/nd280data-numu-cc1pi-xs-on-h2o-2015. 